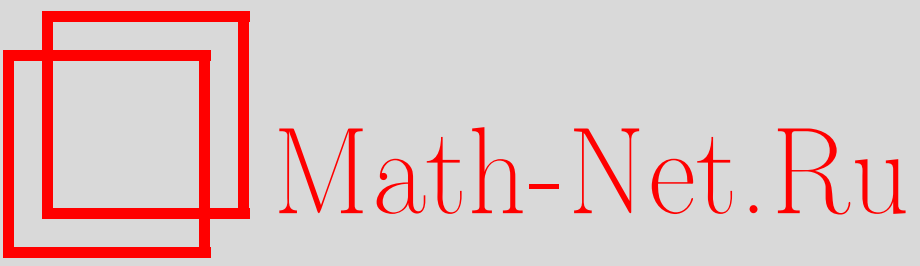

И. А. Дынников, С. П. Новиков, Преобразования Лапласа и симплициальные связности, УМН, 1997, том 52, выпуск 6, 157-158

DOI: https://doi.org/10.4213/rm900

Использование Общероссийского математического портала Math-Net.Ru подразумевает, что вы прочитали и согласны с пользовательским соглашением

http://www.mathnet.ru/rus/agreement

Параметры загрузки:

IP: 54.237 .206 .68

26 апреля 2023 г., 18:37:19 


\title{
ПРЕОБРАЗОВАНИЯ ЛАПЛАСА И СИМПЛИЦИАЛЬНЫЕ СВЯЗНОСТИ
}

\author{
И. А. Дынников, С. П. Новиков
}

В работе [1] были построены аналоги преобразований Лапласа для операторв на дискретной решетке $\mathbb{Z}^{2}$ и указано, что для этого необходимо рассматривать правильную треугольную решетку, порожденную базисными сдвигами $T_{1}, T_{2}$, такими что $\left|T_{1}\right|=\left|T_{2}\right|=\left|T_{1} T_{2}^{-1}\right|$. Каждая вершина взаимодействует с шестью равноудаленными ближайшими соседями. Определение дисктретного преобразования Лапласа и обозначения смотри в [1], [2].

Как замечено в [1], правильная треугольная решетка имеет шесть равноправных базисов, что дает нам три пары взаимнообратных преобразований Лапласа. Слабой эквивалентностью мы назовем преобразование оператора $L \mapsto f L f$, где $f$ - не обращающаяся в нуль функция на решетке $\mathbb{Z}^{2}$. Преобразование Лапласа корректно определено на классах слабой эквивалентности [3].

ПреДЛОЖЕНИЕ 1. Для базисов, отличающихся поворотом на угол $2 \pi / 3$, соответствующие преобразования Лапласа на классах слабой әквивалентности сопряжены $c$ помощью сдвига на вектор решетки $\mathbb{Z}^{2}$.

Таким образом, алгебра преобразований Лапласа на правильной решетке сводится (с точностью до слабой эквивалентности и сдвигов) к двум взаимно обратным преобразованиям. Эти два преобразования можно описать по-другому.

Раскрасим треугольники, составляющие решетку, в шахматном порядке: треуголники вида $\left\langle n, n+T_{1}, n+T_{2}\right\rangle$ покрасим в черньй цвет, а $\left\langle n, n-T_{1}, n-T_{2}\right\rangle$ - в бельй. Пусть $V_{1}, V_{2}, V_{3}-$ пространства функций от вершин, черных треугольников и белых треугольников соответственно. Ближайшими соседями будем называть две вершины, если они соединены ребром, или два треугольника одного цвета, если они имеют общую вершину. Оператором 2-го порядка на $V_{i}$ мы назовем оператор $L: V_{i} \rightarrow V_{i}$, ненулевые внедиагоналшные матричные элементы которого соответствуют парам ближайших соседей.

ПредложениЕ 2. Самосопряженный оператор $L: V_{i} \rightarrow V_{i}$ допускает представление в виде $L=Q_{j i} Q_{j i}^{+}+w$, где $i \neq j, Q_{j i}: V_{j} \rightarrow V_{i}$ - оператор, ненулевые матричные әлементы которого соответствуют в зависимости от $i$ и $j$ либо паре треугольников разного ивета, имеющих общее ребро, либо паре - треугольник и его вершина. Преоразование Лапласа $L \mapsto \widetilde{L}=Q_{j i}^{+} w^{-1} Q_{j i}+1$ совпадает (на классах слабой әквивалентноти) с введеным ранее преобразованием Лапласа на правильной треугольной решетке при надлежащем отождествлении пространств $V_{i} u V_{j}$.

Такое преобразование Лапласа сопоставляет оператору на $V_{i}$ оператор на $V_{j}$, задавая гомоморфииз нулевых уровней: $\psi \mapsto Q_{j i}^{+} \psi$.

Пусть $M$ - произвольная двумерная поверхность с триангуляцией и черно-белой раскраской треугольников, такой, что любые два примькающих по ребру треугольника имеют разные цвета. Пространства $V_{1}, V_{2}, V_{3}$ и отношение ближайшего соседства определим как и ранее.

ПрЕДЛОЖЕНИЕ 3. Если триангуляция поверхности $M$ имеет только четырех- и шестикратные вершинь, то самосопряженные операторы второго порядка на $V_{i}$, $i=1,2,3$, допускают факторизачию и преобразования Лапласа так же, как и в случае правильной решетки на плоскости. Однако, если триангуляиия имеет четырехкратнье вершинь, то факторизации операторов на $V_{2}$ и $V_{3}$ неоднозачны, причем степень неоднозначности определяется числом четырехкратных вершин.

ЗАмечАниЕ. Если триангуляция поверхности $M$ имеет вершины кратности $>6$, то оператор Шрёдингера на черных (белых) треугольниках факторизуется только при выполнении дополнительных условий, причем условия факторизации "через вершины" и “через белые (соотвтетсвенно, черные) треугольники" несовместимы. Подробнее см. в обзоре [4].

Работа первого автора выполнена при поддержке Российского фонда фундаментальных исследований (грант № 96-01-01404). 
Пусть $K$ - произвольный симплициальный комплекс. Симплексы размерности $l$ будем обозначать через $\sigma^{l}$. Зафиксируем некоторые $k<l$. Два симплекса $\sigma_{1}^{l}$ и $\sigma_{2}^{l}$ будем называть ближайшими, если их пересечение содержит некоторый симплекс $\sigma^{k}$. Пусть $L$ - оператор Шрёдингера, действующий на функции от $l$-симплексов так, что взаимодействуют только ближайшие соседи. Векторной факторизачией оператора $L$ c помощью $k$-симплексов мы называем представление $L$ в виде: $L=\sum_{\alpha=1}^{m} Q^{\alpha}\left(Q^{\alpha}\right)^{+}+w$, где $w$ - оператор умножения на функцию, $Q^{+}=\left(\left(Q^{1}\right)^{+}, \ldots,\left(Q^{m}\right)^{+}\right)$- оператор на функциях от $l$-симплексов со значениями в $m$-вектор-функциях от $k$-симплексов:

$$
\left(Q^{+} \psi\right)_{\sigma^{k}}^{\alpha}=\sum_{\sigma^{l} \supset \sigma^{k}} b_{\sigma^{k}: \sigma^{l}}^{\alpha} \psi_{\sigma^{l}} .
$$

Факторизацию оператра $L$ назовем специальной, если $w=$ const. Векторной факторизации соответствует преобразование типа Лапласа: $L \mapsto \widetilde{L}=Q^{+} w^{-1} Q+1$, которое сопоставляет оператору $L$ оператор $\widetilde{L}$ на вектор-фукнциях от $k$-симплексов.

Аналогичным образом можно определить факторизацию оператора, действующего на $l$-симплексах, с помощью $k$-симплексов в случае $k>l$ : будем считать два $l$-симплекса ближайшими соседями, если они содержатся в некотором $k$-симплексе.

В качестве примера рассмотрим решетку правильных тетраэдров в $\mathbb{R}^{3}$ : в качестве базисна $T_{1}, T_{2}, T_{3}$ решетки $\mathbb{Z}^{3}$ возьмем векторы, соединяющую некоторую вершину правильного тетраэдра в $\mathbb{R}^{3}$ с остальными вершинами. Рассмотрим комплекс $K$, составленный из тетраэдров, полученных из данного всевозможными сдвигами на векторы решетки $\mathbb{Z}^{3}$. Пусть оператор $L$ действует на решетке $\mathbb{Z}^{3}$, причем взаимодействуют только вершины, соединенные ребром.

ПредЛожениЕ 4. СКалярная факторизаиия оператора L с помощью 3-симплексов комплекса $K$ возможна тогда и только тогда, когда для каждого тетраэдра из $K$ произведения коэффичиентов связности, сидящих на противополежсащих ребрах, совпадают. Специальная т-векторная факторизачия L с помощью 3-симплексов возмоэсна при любом $m>1$, и притом неоднозначна.

Отыскание основных состояний операторов Шрёдингера на $l$-симплексах в ряде случаев сводится к решению системы уравнений $Q^{+} \psi=0$, где оператор $Q^{+}$имеет вид (1). Такую систему уравнений мы назовем симплициальной связностью, если она удовлетворяет требованияю невырожденности и требованию локальности, определение которых смотри в обзоре [4]. Условия локальной совместности такой системы приводит к дискретным аналогам кривизны.

Примером симплициальной связности являются уравнения треугольника, рассмотренные в [3]. Их обобщением является уравнение тетраэдров в $\mathbb{R}^{3}$ :

$$
Q_{1} \psi=0, \quad Q_{2}^{+} \psi=0, \text { где } Q_{1,2}=1+x_{1,2} T_{1}+y_{1,2} T_{2}+z_{1,2} T_{3} .
$$

ПреДЛОЖЕНИЕ 5. Условием полной локальной совместности системы (2) является соотношение $\left(Q_{1}-1\right)\left(Q_{2}^{+}-1\right)-1=f\left(\left(Q_{2}^{+}-1\right)\left(Q_{1}-1\right)-1\right)$, где $f$ - некоторая функция на $\mathbb{Z}^{3}$.

Другие примеры симплициальных связностей и дискретных аналогов преобразования Лапласа см. в [4].

\section{СПИСОК ЛИТЕРАТУРЫ}

[1] Novikov S. P. Appendix I in Novikov S. P., Veselov A. P. // Trans. Amer. Math. Soc. Ser. 2. 1997. V. 179. Р. 109-132. [2] Новиков С. П. // УМН. 1997. Т. 52. № 1. С. 225-226. [3] Novikov S.P. Difference 2D Schrödinger operators: Algebraic symmetry and exact solvability // Preprint. Paris: Univ. Paris-VII, April 1997. [4] Новиков С. П., Дынников И. А. // УMH. 1997. T. 52. № 5. C. 175-234.

Московский государственньй университет им. М. В. Ломоносова; University of Maryland at College Park 